\title{
Polymer micro-lenses as a long-coupling-distance interfacing layer in the low-cost optical coupling solution between optical fibers and photonic integrated waveguide circuits
}

\author{
Andrzej Kaźmierczak ${ }^{1 *}$, Mateusz Słowikowski ${ }^{1,2}$, Krystian Pavłov², Maciej Filipiak ${ }^{2}$ and Ryszard Piramidowicz ${ }^{1}$ \\ ${ }^{1}$ Institute of Microelectronics and Optoelectronics, Warsaw University of Technology, Koszykowa 75, \\ 00-662 Warszawa, Poland \\ ${ }^{2}$ CEZAMAT, Warsaw University of Technology, Poleczki 19, 02-822 Warsaw, Poland
}

Received December 12, 2019; accepted December 31, 2019; published December 31, 2019

\begin{abstract}
We present a low-cost scheme for the non-permanent optical signal coupling for prospective application in single use photonic integrated chips. The proposed scheme exploits the use of polymer kinoform microlenses. The feasibility of the proposed solution is demonstrated by experimental investigation of an optical signal coupling from single mode optical fiber (SMF) to the test structure of $\mathrm{Si}_{\mathrm{x}} \mathrm{N}_{\mathrm{y}}$ integrated waveguide.
\end{abstract}

Nowadays the main photonic integrated circuit (PIC) technologies, including InP [1], SOI and SiN [2], have grown up to the level where most of the simple integrated optical structures (e.g. waveguides, couplers, photodiodes, modulators etc.) have reached their maturity. Consequently, these components may be treated as fundamental building blocks in generic PIC technology. This gives us greater freedom in designing more complex PICs (similarly in designing electronic ICs) in order to meet the needs of the market. As a result, PICs can be ordered commercially at several PIC foundries.

The photonic circuit packaging, however, is still an issue. One of the vital problems in this field is providing an efficient and low-cost solution for an optical coupling to PICs, particularly evident in the case of single-use disposable PICs (e.g. optical sensor chips), where the use of a standard approach to permanently attach optical fibers to photonic circuits is too expensive. In this paper we discuss the possibility of using polymer micro-lenses as a low-cost optical interface, providing long-distance separation between the input waveguides of PIC and optical fibers while coupling the optical signal.

The fundamental difficulty in coupling the optical signal from a single-mode fiber (SMF) to the integrated photonic waveguide is the substantial difference of its crosssections. Typically, the diameter of an SMF core ranges from 5 to $9 \mu \mathrm{m}$, while the integrated waveguides usually are up to $2 \mu \mathrm{m}$ wide and few hundred nanometers high. This leads to obvious geometrical mismatch. With the

\footnotetext{
*E-mail: a.kazmierczak@imio.pw.edu.pl
}

development of PIC technologies a number of solutions were proposed, mainly including a fiber-to-edge-of-thechip coupling with spot-size converters (SSC) [3-4] or a vertical coupling with grating couplers (SGC) [5-6] both adapting an effective waveguide mode profile to the mode profile of SMF. Further, lensed or tapered fibers can be used for the purpose of an optical signal coupling. While all types of solutions provide considerable coupling efficiencies, they all require precise alignment between the SMF and the input waveguide of PIC (typically with a precision better than $\pm 100 \mathrm{~nm}$ ). This limitation is far beyond the typical precision of packaging technologies such as injection molding ( $\pm 20 \mu \mathrm{m}$ precision). It can be, however, relatively easy solved by using alignment stages offering sufficient precision.

In the case of an optical sensor system, the single use of a disposable optical chip can be placed in the readout device equipped with electro-mechanical actuators providing optical-coupling fine-tuning with sufficient precision. Unfortunately, usually the end of an optical fiber has to be placed very closely to the chip facet $(<<1 \mu \mathrm{m})$, which brings a substantial risk of physical contact between the fiber and the PIC, possibly leading to the damage of one or another.

When the lens is placed between the end of an optical fiber and the chip edge it can act as an interface between PIC and SMF, enhancing coupling efficiency and providing necessary spacing between them [7-9].

Typically, sensor chips include a number of optical sensors, each equipped with separate input and output waveguides, therefore a number of coupling interfaces are needed. This cannot be addressed by a multitude of typical (e.g. 0.5 inch diameter) lenses or microscope objectives. However, an array of properly chosen microlenses assembled with several SMFs may offer an interesting solution for such a need.

In subsequent sections of this paper the use of polymer kinoform microlenses for optical coupling between SMF and PIC will be discussed in detail, showing some preliminary results obtained. 
The size of a microlens used as an interface between PIC and SMF is a compromise between the easiness of its use (requiring as large a lens as possible) and the minimal separation of the input waveguides in PIC (set by the lens diameter) therefore lenses of two diameters were designed, namely $1 \mathrm{~mm}$ and $2 \mathrm{~mm}$. The designed focal lengths of microlenses were set to $1.5 \mathrm{~mm}$ and $3.0 \mathrm{~mm}$, respectively.

In order to determine the lens shape, the simplest form of lens maker equation was used namely:

$$
R=f \cdot(n-1)
$$

where $R$ is the radius of curvature of the lens, $f$ is the focal distance and $n$ is the refractive index of the lens material (set to 1.5 for calculation simplicity). The obtained result was then sliced, using the modulo function into $2 \mu \mathrm{m}$ height areas, leading to $3^{\text {rd }}$ order kinoform lenses design.

The designed microlenses were fabricated at WUT premises in CEZAMAT laboratory. The pattern was manufactured using grayscale electron beam lithography a technique that creates three-dimensional features in a single exposure by modulating an electron dose in each spot or sub-region. After polymer development, the desired three-dimensional structure was revealed. However, grayscale lithography had its limitations - resist films thicker than $10 \mu \mathrm{m}$ could only be manufactured by multiple spin-coating, which negatively affects film uniformity. Moreover, the surface roughness of the final structure increased with modulation depth, which affected optical performance. In order to achieve chosen lens diameters and desired focal lengths, spherical kinoform lenses were designed and manufactured, as their height can be easily controlled.

The $700 \mu \mathrm{m}$-thick glass substrate was spin-coated with a $3.5 \mu \mathrm{m}$ layer of PMMA950 positive electron beam resist and $40 \mathrm{~nm}$ of AR-PC 5091 Electra conductive polymer.

The PMMA950 polymer has a refractive index of 1.4956 for a wavelength of $657 \mathrm{~nm}$, according to its producer. The lens pattern with $2 \mu \mathrm{m}$ depth modulation was exposed using a JEOL JBX9300FS electron beam lithography tool. The conductive layer was removed in deionized water and the pattern was developed using a 1:1 MIBK-IPA mixture. The lenses were then reflown at $110^{\circ} \mathrm{C}$ for $100 \mathrm{sec}$. to further reduce surface roughness.

Micrographs of fabricated lenses are shown in Fig. 1.

A proper circular shape of lenses can be noticed, with a number of rings. In the case of a $2 \mathrm{~mm}$ diameter lens a slight, a cross shape imperfection is noticeable. It was caused by stitching a number of e-beam writing fields necessary to write such a large structure. Some shadow effects can be noticed in micrographs, possibly affecting the efficiency of the outer area of the lenses. The SEM micrograph shows the flatness of the lens and proper shape of the rings, as only some imperfections smaller than $1 \mu \mathrm{m}$ in the shape of rings can be observed.

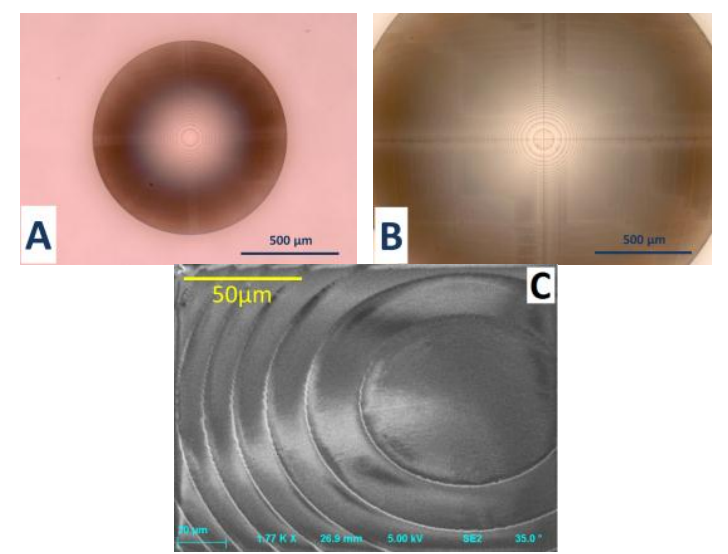

Fig. 1. Optical micrographs of PMMA lenses of $1 \mathrm{~mm}$ (A) and $2 \mathrm{~mm}$ (B) in diameter and the SEM image of a $1 \mathrm{~mm}$ diameter lens (C).

The operation of fabricated lenses was validated by generating a quasi parallel beam using these lenses. The experiment was conducted by inserting the optical signal from a red light laser (658 nm wavelength) with an SMF located at the focal point of the lens by means of a 3D tunable translation stage. The obtained beam profile was measured with a Thorlabs BC 106 - VIS beam profiler located approximately within a $20 \mathrm{~cm}$ distance from the lens. The obtained results are depicted in Fig. 2.
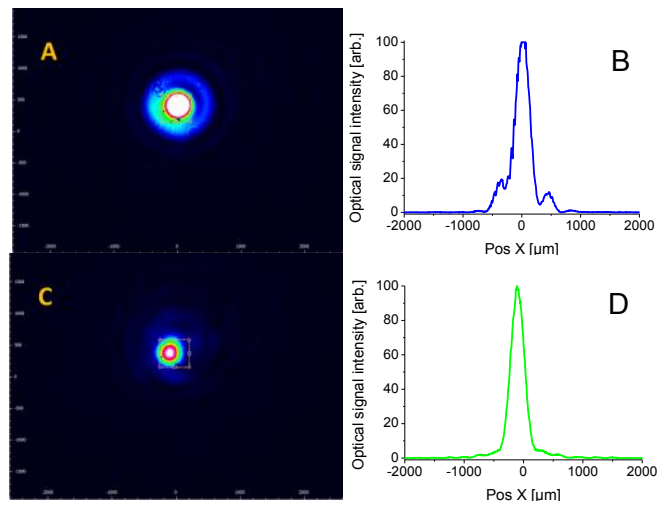

Fig. 2. Images of quasi parallel-beams(A), (C) and the profiles of these beams(B), (D) generated by using kinoform PMMA lenses having diameters of $1000 \mu \mathrm{m}$ and $2000 \mu \mathrm{m}$ respectively.

The usefulness of fabricated microlenses for optical coupling was evaluated by coupling the optical signal from $\mathrm{SMF}$ to the test structure of $\mathrm{Si}_{\mathrm{x}} \mathrm{N}_{\mathrm{y}}$ integrated waveguide having a rectangular cross section $(320 \mathrm{~nm}$ high and $2.0 \mu \mathrm{m}$ wide) via investigated microlens.

The measurement was conducted using a red light source (658 nm wavelength). Both the PIC (containing $\mathrm{Si}_{\mathrm{x}} \mathrm{N}_{\mathrm{y}}$ integrated waveguides) and optical SMF, pigtailing laser diode, were placed in 3D translation stages, providing 
alignment precision better than $\pm 100 \mathrm{~nm}$ in each axis. The glass plate containing the investigated microlenses was fixed firmly in the holder. Then the output of an SMF was placed in front of the lens at the distance more than 2 times exceeding its focal length. The position of the SMF tip was then aligned in order to produce the spot at the input facet of PIC. The position of the input waveguide of PIC was subsequently adjusted in order to optimize the optical signal coupling. In order to evaluate the coupling efficiency, the light leaving the PIC via the output waveguide was collected with a microscope objective and coupled to the optical power meter. The experiment is shown in Fig. 3.
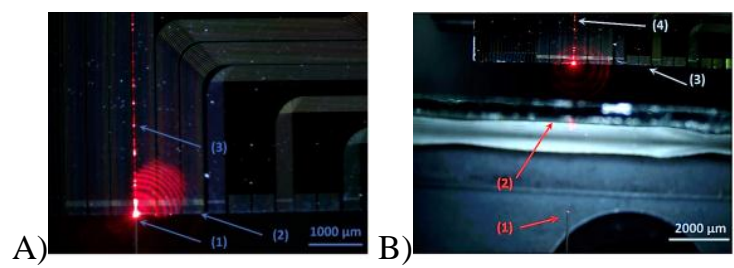

Fig. 3. Direct optical signal coupling between $\mathrm{SMF}$ and integrated $\mathrm{Si}_{\mathrm{x}} \mathrm{N}_{\mathrm{y}}$ (A) and coupling via kinoform microlens (B). (1) SMF tip, (2) glass substrate with microlenses (3) PIC edge, (4) integrated waveguide guiding light.

The first obtained results of optical coupling efficiency were relatively low and the measured output power was $9.0 \mathrm{~dB}$ (in the case of a $1 \mathrm{~mm}$ diameter lens) up to $9.4 \mathrm{~dB}$ (in the case of a $2 \mathrm{~mm}$ diameter lens), being smaller than those obtained with a direct coupling of SMF. The possible cause of this low coupling efficiency is the influence of a glass substrate, causing additional reflection and expanding the outcoming beam waist. In the case of prospective sensor application, the low injected power, however, is not a crucial issue and power loss budgets of even $-30 \mathrm{~dB}$ could be accepted.

Nonetheless, the introduction of microlenses to the coupling scheme allowed achieving a significant coupling distance (exceeding $1.3 \mathrm{~mm}$ between the glass substrate of the lens array to the PIC facet). Such a distance would certainly ensure safe execution of automated alignment between the sensor chip and the readout unit.

We have demonstrated that the introduction of polymer kinoform microlenses as an interfacing element for optical coupling between integrated waveguides and SMFs provides a significant geometrical separation between these elements. Further work will be carried out towards increasing coupling efficiency either by developing lenses of different focal length or constructing an assembly of two microlenses, the former producing quasi-parallel beam and the latter focusing it to the small-size spot at the input waveguide. Finally, the microlenses will be fabricated in the form of a lens array and assembled with a multitude of SMFs in order to test simultaneous optical coupling to several optical waveguides. It is envisaged that the analyzed lenses will be developed into a multichannel low-cost optical interface between SMFs and PICs as their final configuration lenses arrays can be fabricated using low-cost hot embossing technology.

This work was partially supported by the Polish National Science Centre in the framework of Miniatura I program 2017/01/X/ST7/01314

\section{References}

[1] M. Smit et al., Semiconductor Science and Technology 29(8), 083001 (2014).

[2] R. Baets et al., Optical Fiber Communication Conference, OSA Technical Digest (online) (Optical Society of America, 2016), paper Th3J.1.

[3] K. Shiraishi et al., Appl. Phys. Lett. 91, 141120 (2007).

[4] Y. Sobu et al., IEEE Photonic Society 24th Annual Meeting, 469-470, (2011).

[5] F. Van Laere et al., J. Lightwave Technology 25(1), 151 (2007).

[6] A. Kaźmierczak et al., Opt. Eng. 48, 014401 (2009).

[7] M. Rossi et al., Appl. Opt. 34, 2483 (1995).

[8] M. Prasciolu et al., Japanese J. of Applied Physics 42, 4177 (2003).

[9] F. Schiappelli et al., Microelectronic Engineering 73-74, 397 (2004). 